\title{
Research Paper: Comparison of Embryo Development and Pregnancy Rates in Continuous Single and Sequential Media Cultures of Sibling Embryos
}

Akiko Takashima ${ }^{1}$, Fuminori Kimura ${ }^{*}$, Kazumi Kishida ${ }^{1}$, Mika Izuno ${ }^{1}$, Kimiko Hirata ${ }^{1}$, Akie Takebayashi ${ }^{1}$, Tsukuru Amano ${ }^{1}$, Shunichiro $^{1}$ Tsuji $^{1}$, Shoji Kaku', Tesuo Onoa ${ }^{1}$, Kentaro Takahashi ${ }^{2}$, Takashi Murakmai ${ }^{1}$

1. Department of Obstetrics and Gynecology, Shiga University of Medical Science, Setatsukinowacho, Otsu, Shiga, Japan.

2. Department of Community Perinatal Medicine, Shiga University of Medical Science, Setatsukinowacho, Otsu, Shiga, Japan.

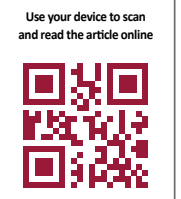

Citation: Takashima A, Kimura F, Kishida K, Izuno M, Hirata K, Takebayashi A. et al. Comparison of Embryo Development and Pregnancy Rates in Continuous Single and Sequential Media Cultures of Sibling Embryos. Journal of Advanced Medical Sciences and Applied Technologies. 2017; 3(3):147-154. https://doi.org/10.32598/jamsat.3.3.147

https://doi.org/10.32598/jamsat.3.3.147

Article info:

Received: 01 May 2017

Accepted: 26 Jul. 2017

\section{Keywords:}

Embryo development, In-vitro fertilization, Single media culture, Sequential media culture

\section{A B STRACT}

Objectives: Embryo development and pregnancy rates for In Vitro Fertilization (IVF) in continuous single medium culture or sequential media culture of sibling embryos were evaluated.

Matrials \& Methods: Patients who underwent retrieval of $\geq 8$ oocytes from June 2013 to March 2014 were enrolled. All embryos were cultured for at least 5 days and the formation rate and quality of embryos were compared between the groups.

Results: No significant differences were observed between 2 culture media systems regarding the formation rate and quality of early cleavage-stage embryos, although blastulation rate and quality were better with single medium. Clinical pregnancy rate and ongoing pregnancy rate per blastocyst transfer did not differ between the groups.

Conclusion: These results suggested that a single continuous medium was as good as or better than sequential media to achieve pregnancy in IVF and embryo transfer.

\section{Introduction}

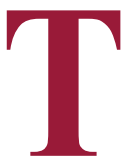

he environment surrounding an embryo during In Vitro Fertilization (IVF) is determined by culture conditions such as the composition of the culture medium, the temperature, and carbon dioxide concentration in incubator $[1,2]$. The composition of culture media is a main concern regarding the success of IVF and embryo transfer. Two opposing views were put forward regarding the ideal composition of culture media: "back to nature" and "embryo free choice/single culture medium" $[3,4]$. Based on the concept of "back to nature", sequential media were developed to mimic the natural sequence of conditions encountered when the embryo lies in the fallopian tube or uterine cavity [5, 6]. Different substances required at each moment in the fallopian tube or uterine cavity is contained in sequen-

* Corresponding Author:

Fuminori Kimura, MD, PhD

Address: Department of Obstetrics and Gynecology, Shiga University of Medical Science, Seta-Tsukinowa-cho Otsu Shiga, Japan.

Tel: +81 (77) 5482267

E-mail: kimurafu@belle.shiga-med.ac.jp 
tial media to optimize embryo quality. One such requirement is the energy supply for the embryo, which changes during the development of the embryo [7-9].

On the other hand, based on the concept of "embryo free choice/single culture medium", the embryo is cultured in a single medium that is not renewed. This single medium contains all the components needed for embryo development. Advocates of this concept assert that the evidence does not support a need for sequential media for embryo development in vitro, and the embryo will utilize whatever it requires [10-12]. The transfer of embryos to a new dish and renewal of the culture medium at intervals were suggested to expose embryos to rapid environmental change and increased risk of phototoxicity, although it can prevent the exposure of embryos to the potential accumulation of ammonium from amino acids metabolism [7, 13, 14].

Which culture condition is required to achieve the best embryo quality remains contentious [7, 13]. Optimal culture media for embryo quality were reported in either sequential or single media, and other reports showed no difference in the quality of cleavage-stage embryo and blastocyst between a single medium and sequential media. These various results might be due to variations in the settings of the studies. However, 2 recent reports showed the advantage of continuous single medium culture.

Reed et al. randomized fertilized oocytes into 2 commercial media and revealed the superiority continuous single medium culture based on a greater number of available blastocysts [15]. However, they used an average of 2.1 blastocysts for embryo transfer with transfer of multiple embryos selected from both media in 22 of 44 transfers $(50.0 \%)$. Thus, the actual implantation rate of embryos from each medium could not be determined.

Sepulveda et al., showed that in vitro development rates on the days 3 and 5 and implantation rates were significantly greater in the single medium with medium renewal on the day 3 , compared with a sequential media system for development of human embryos to the blastocyst stage and subsequent pregnancy outcomes [16]. However, oocytes in their study were obtained not from infertile patients, but rather from young healthy donors. As the mean age of patients undergoing IVF is rising in developed countries, randomized evaluations for sibling oocytes should be performed for infertile patients as well as young donors, as the metabolic activity of eggs may differ between them.

The present study aimed at clarifying the optimal culture conditions between sequential and single media, by comparing embryo development at the cleavage and blastocyst stages after randomized division of sibling oocytes from infertile patients as well as comparing subsequent pregnancy rates by single blastocyst transfer under identical hormone-replacement conditions for endometrial preparation.

\section{Materials and Methods}

In the present study, only the standardized Assisted Reproductive Technology (ART) procedures were employed. The study was concerned with the development of cultured embryos and outcomes of pregnancy between 2 commercially available media that are commonly used. No other additional experimental treatments were used. Institutional review board approval was therefore not applicable regarding the allocation of embryos to 2 different culture media $[16,17]$.

Patients treated with IVF at Shiga University of Medical Science, Japan, from October 2013 to June 2015 were registered to the study when the number of oocytes retrieved was 8 or more after obtaining written consent to oocyte retrieval and verbal explanation of division of embryos into 2 different culture media systems followed by the consent documented by doctors on clinical record. In the Gonadotropin-Releasing Hormone (GnRH) agonist protocol, patients underwent pituitary down regulation with $600 \mathrm{mg} /$ day of GnRH agonist (Suprecur ${ }^{\mathbb{R}}$; Mochida Pharmaceutical, Tokyo, Japan) for over 3 months (ultra-long protocol), from the mid-luteal period of the previous menstrual cycle (long protocol) or from the 3rd day of menstruation (short protocol).

Follicle-stimulating hormone (Folyrmon-P; Fuji Pharma, Tokyo, Japan) and/or human menopausal gonadotropin (HMG INJECTION TEIZO; ASKA Pharmaceutical, Tokyo, Japan) was used for follicular stimulation in doses of 150 to $450 \mathrm{IU} /$ day. Initial dose and type of gonadotropin were determined by the number of visible antral follicles and serum follicle-stimulating hormone level at the start of controlled ovarian stimulation. Follicular growth was observed by transvaginal ultrasound every 1 to 3 days.

Intramuscular administration of 10,000 units of Human Chorionic Gonadotropin (hCG) was performed when 2 or more follicles reached $18 \mathrm{~mm}$ in diameter. In the antagonist protocol, 150 to $450 \mathrm{IU} /$ day of gonadotropin was administered similar to that of the agonist group from the $3^{\text {rd }}$ day of menstruation. After a dominant follicle reached $14 \mathrm{~mm}$ in diameter, $0.25 \mathrm{mg}$ of GnRH antagonist (Cetrotide $^{\circledR}$; Nippon Kayaku, Tokyo, Japan) was applied every day until the day of hCG injection. When 2 or more folli- 
cles reached $18 \mathrm{~mm}$ in diameter, 10,000 units of hCG was injected intramuscularly. Oocyte pickup was performed 35 hours after hCG administration based on a standard method. After oocyte retrieval, cumulus-oocyte complexes were washed to strip excessive cumulus cells and cultured in 500 to $1000 \mathrm{~mL}$ of Universal IVF Medium ${ }^{\circledR}$ (Origio, Måløv, Denmark) for 2 to 4 hours. Semen was collected by masturbation from the patients' husbands. Motile spermatozoa were separated from seminal plasma by centrifugation through $80 \%$ isolate buffer (PureCeption $^{\mathrm{TM}}$ Sperm $^{\circledR}$; Origio) for 20 minutes at $500 \mathrm{~g}$. Sperms were, then, washed with Universal IVF Medium ${ }^{\circledR}$.

For oligospermic samples, sperms were washed and resuspended in varying amounts of Universal IVF Medi$\mathrm{um}^{\circledR}$, depending on the initial concentration and motility. Then, spermatozoa were extracted from testicular sperm and collected for Intra-Cytoplasmic Sperm Injection (ICSI) after thawing the testicular tissue using a standard method. For conventional insemination, approximately 100,000 spermatozoa were placed in $500 \mathrm{~mL}$ of Universal IVF Medium ${ }^{\circledR}$ containing 3 oocytes. The ICSI procedure was performed by a standard method. After insemination, all oocytes were cultured in $30-\mathrm{mL}$ drops of Universal IVF Medium ${ }^{\circledR}$ for 16 to 20 hours at $37^{\circ} \mathrm{C}$. Embryo culture media were prepared according to the instructions of the manufacturer.

$\mathrm{O}_{2}$ and $\mathrm{CO}_{2}$ concentrations in incubator were set to $5.0 \%$ each for both media. In the morning of the day 1 after fertilization, sibling zygotes with 2 pronuclei were randomly divided into either sequential media $\left(\mathrm{ECM}^{\mathrm{TM}}\right.$ medium and multiBlast; IrvineScientific, Santa Ana, CA) or a continuous single medium (global $^{\circledR}$ total $^{\circledR}$, LIFEGLOBAL GROUP, Guilford, CT). Both media were supplemented with $10 \%$ v/v Synthetic Serum Substitute (SSS). On the day 3, embryos cultured in $\mathrm{ECM}^{\mathrm{TM}}$ with $10 \% \mathrm{SSS}$ were transferred into $30-\mu \mathrm{L}$ droplets of multiblast with $10 \%$ SSS. Embryos in global total ${ }^{\circledR}$ with $10 \%$ SSS were cultured from the day 1 to the day 5 or 6 without medium renewal.

On the day 2 or 3 , embryos were evaluated for cell number, blastomere size, and fragmentation according to the Veeck classification [18]. Grades 1 and 2 embryos were defined as good when there were 4 to 6 blastomeres on the day 2 or 8-12 on the 3rd day of culture. Patients treated by early cleavage stage embryo transfer were excluded from the present study. Thus, in this study all embryos were cultured and observed until the day 5. On days 5 and 6 , embryos were estimated and frozen when embryos were developed to fully expanded blastocyst. Blastocysts were estimated by size, Inner Cell Mass (ICM), and trophectoderm according to the Gardner classification. Blastocysts with grade 3BB or better, expansion grade scale score of 3 or more, A or B inner cell mass, and A or B trophectoderm were defined as good.

Blastocysts were frozen according to the instructions of Kitazato BioPharma (Tokyo, Japan). Frozen-thawed blastocyst transfer was performed for all patients under an identical endometrium preparation with supplementation with a transdermal estradiol patch (Estrana ${ }^{\mathbb{R}}$; Hisamitsu Pharmaceutical, Tokyo, Japan) from the 2nd or 3rd day of the cycle date and dydrogesterone (Duphaston $^{\circledR}$; ABBOTT JAPAN, Tokyo, Japan) from the 14th or 15 th day of the cycle. Blastocyst transfer was performed 6 days after supplementation with dydrogesterone. Frozen blastocysts were thawed on the morning of the day of embryo transfer in accordance with the instructions of Kitazato BioPharma (Tokyo, Japan). Thawed blastocysts were cultured in the same brand medium used for culture before freezing the blastocysts.

PhyCon IVF catheter (Fuji Systems, Tokyo, Japan) or Supported Embryo Transfer catheter (Kitazato BioPharma) was used for blastocyst transfer. All transfers were performed under transabdominal ultrasonography monitoring. Approximately 14 days after transfer, pregnancy was determined based on urinary hCG levels. Transvaginal ultrasonography was used to detect gestational sacs from 14 to 21 days after transfer. When a gestational sac was detected, the patient was defined as pregnant.

The developmental rates of cleavage embryo, good cleavage embryo, blastocyst and good blastocyst for zygotes were estimated and clinical pregnancy rates and ongoing pregnancy rates from each culture system (continuous single culture group or sequential media culture group) were also evaluated. As patients differed in age, clinical and ongoing pregnancy rates were also compared only in patients with 33 years old or above. Statistical analysis was performed using Chi-square test or the Fisher exact test, as appropriate. Patient characteristics regarding the outcomes of embryo transfer were compared using the Student $t$ test.

\section{Results}

Patients were treated at Shiga University of Medical Science, Japan, from October 2013 to June 2015. The study included a total of 42 cycles from 40 infertile females treated with IVF due to refractory infertility (age range 29 to 44 years; Mean(SD) $=35.8 \pm 3.9$ years) (Tables 1 and 2). GnRH agonist or GnRH antagonist was used in 36 cycles and 6 cycles, respectively. A total of 603 oocytes were collected. Conventional IVF, ICSI, TESE-ICSI, and 


Type of insemination
Conv: 28
ICSI: 12
TEST-ICSI: 1
Split: 1

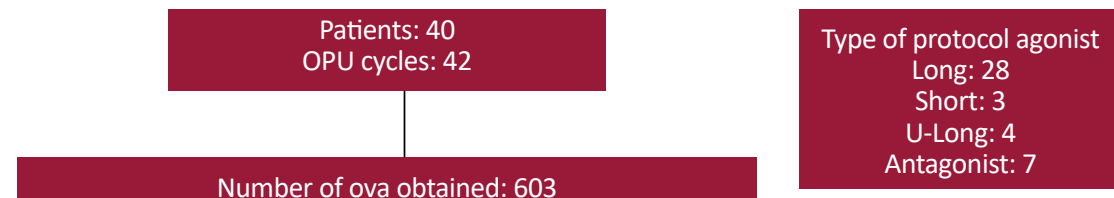

Figure 1. Flow diagram of the study comparing continuous single versus sequential media culture

The study investigated 42 cycles in 40 infertile females treated by IVF due to refractory infertility. Long-short-, or ultra-long protocol with GnRH agonist or GnRH antagonist protocol was used in 28, 3, 4, and 7 cycles, respectively. A total of 603 oocytes were collected. Conventional IVF, ICSI, TESE-ICSI, and split to conventional IVF and ICSI were used for fertilization in 28, 12, 1, and 1 cycle(s), respectively. The resulting 348 fertilized embryos with 2 pronuclei were randomized (same number of embryos for each group as much as possible) into sequential media (162 embryos) or single medium (186 embryos).

split were used for fertilization in 28, 12, 1, and 1 cycle(s), respectively (Figure 1). In 42 cycles of IVF, the 348 fertilized embryos (zygotes) with 2 pronuclei were randomized (same number of embryos for each group as much as possible) into the sequential culture media group (162 embryos) and the continuous single culture group (186 embryos) (Figure 1). Percentages of cleavage embryos, good cleavage embryos, blastocysts, and good blasto- cysts per zygote were 95.1\%(154/162), 34.0\%(55/162), $33.3 \%(54 / 162)$, and $14.2 \%(23 / 162)$ in the sequential culture media group, and 96.8\%(180/186), 33.3\%(62/186), $43.5 \%(82 / 186)$, and $23.6 \%(45 / 186)$ in the continuous single culture group, respectively (Table 3 ).

Although no significant difference was observed regarding the proportion of cleavage embryos $(\mathrm{P}=0.49)$

Table 1. Patients' characteristics in the study

\begin{tabular}{cc}
\hline \multicolumn{1}{c}{ Patients' Characteristics } \\
\hline Number of patients & 40 \\
\hline Number of treatment cycles & 42 \\
Patients mean \pm SD age (year) & $35.8 \pm 5.8$ \\
Age range & $29-44$ \\
Gravidity & $0.71 \pm 0.89$ \\
Parity & $0.26 \pm 0.49$ \\
\hline
\end{tabular}

SD: Standard Deviation

JAMSAT

Table 2. Cause of infertility

\begin{tabular}{cc}
\hline Cause of Infertility & Number of Patients \\
\hline Male factor & 15 \\
\hline Tubal factor & 7 \\
\hline Endometriosis & 9 \\
\hline Ovulation disorder & 3 \\
\hline Fertilization disorder & 3 \\
\hline Unknown & 9 \\
\hline
\end{tabular}

Causes were overlapped in some patients.

JAMSAT 
Table 3. Embryo development at cleavage stage and blastocyst stage

\begin{tabular}{|c|c|c|c|}
\hline & Sequential & Single & $\mathbf{P}$ \\
\hline Cleavage rate & $95.1 \%(154.162)$ & $96.8 \%(180.186)$ & N.S. \\
\hline Good cleavage rate & $34.0 \%(55.162)$ & $33.3 \%(62.186)$ & N.S. \\
\hline Blastulation rate & $33.3 \%(54.162)$ & $44.1 \%(82.186)$ & $<0.05$ \\
\hline Good blastulation rate & $14.2 \%(23.162)$ & $24.2 \%(45.186)$ & $<0.03$ \\
\hline
\end{tabular}

JAMSAT

and good cleavage embryos $(\mathrm{P}=0.91)$, a significantly greater proportion of embryos developed to blastocysts $(\mathrm{P}=0.048)$ and good blastocysts $(\mathrm{P}=0.021)$ in the continuous single culture group than in the sequential media culture group. A total of 42 blastocysts (12 from the sequen- tial media culture group, 32 from the continuous single culture groups) were used for single blastocyst transfer.

Gravidity, Body Mass Index (BMI), and basal Follicle Stimulating Hormone (FSH) did not differ between the sequential media culture and continuous single culture

Table 4. Characteristics of patients enrolled for single blastocyst transfer

\begin{tabular}{|c|c|c|c|}
\hline & Sequential ( $n=12)$ & Single $(n=32)$ & $\mathbf{P}$ \\
\hline $\begin{array}{l}\text { Mean } \pm S D \text { age (year) } \\
\text { Age range (year) }\end{array}$ & $\begin{array}{c}37.9 \pm 2.7 \\
(33-44)\end{array}$ & $\begin{array}{c}35.3 \pm 4.0 \\
(29-44)\end{array}$ & $<0.03$ \\
\hline $\mathrm{BMI}\left(\mathrm{kg} / \mathrm{m}^{2}\right)$ & $\begin{array}{c}21.8 \pm 2.9 \\
(18.1-28.8)\end{array}$ & $\begin{array}{c}21.2 \pm 2.6 \\
(17.1-28.8)\end{array}$ & N.S. \\
\hline $\mathrm{FSH}(\mathrm{IU} / \mathrm{mL})$ & $\begin{array}{l}7.9 \pm 2.4 \\
(5.2-14.7)\end{array}$ & $\begin{array}{c}7.8 \pm 1.3 \\
(5.2-10.3)\end{array}$ & N.S. \\
\hline Gravity & $\begin{array}{c}0.83 \pm 0.90 \\
(3-0)\end{array}$ & $\begin{array}{c}0.59 \pm 0.86 \\
(3-0)\end{array}$ & N.S. \\
\hline Parity & $\begin{array}{c}0.58 \pm 0.64 \\
(2-0)\end{array}$ & $\begin{array}{c}0.16 \pm 0.36 \\
(1-0)\end{array}$ & $<0.03$ \\
\hline $\begin{array}{l}\text { Period of fertility treat- } \\
\text { ment (month) }\end{array}$ & $\begin{array}{c}17.3 \pm 13.8 \\
(3-96)\end{array}$ & $\begin{array}{c}24.9 \pm 20.9 \\
(3-96)\end{array}$ & N.S. \\
\hline Cause of infertility & $\begin{array}{l}\text { Male factor: } 5 \\
\text { Tubal factor: } 3 \\
\text { Endometriosis: } 1 \\
\text { Ovulation disorder: } 1 \\
\text { Fertilization disorder: } 0 \\
\text { Unknown: } 4\end{array}$ & $\begin{array}{l}\text { Male factor: } 10 \\
\text { Tubal factor: } 6 \\
\text { Endometriosis: } 7 \\
\text { Ovulation disorder: } 2 \\
\text { Fertilization disorder: } 1 \\
\text { Unknown: } 5\end{array}$ & \\
\hline Fertilization method & $\begin{array}{l}\text { Conventional: } 9 \\
\text { ICSI: } 2 \\
\text { TESE-ICSI: } 0 \\
\text { Split: } 1\end{array}$ & $\begin{array}{l}\text { Conventional: } 22 \\
\text { ICSI: } 9 \\
\text { TESE-ICSI: } 0 \\
\text { Split: } 1\end{array}$ & \\
\hline
\end{tabular}

SD: Standard Deviation; BMI: Body Mass Index; FSH: Follicle-Stimulating Hormone

JAMSAT

Table 5. Outcomes in terms of pregnancy rate and ongoing pregnancy rate

\begin{tabular}{cccc} 
& Sequential Culture Media $(\mathbf{n}=\mathbf{1 7})$ & Single Culture Media $(\mathbf{n = 3 8})$ & $\mathbf{P}$ \\
\hline Pregnancy rate & $50.0 \%(6 / 12)$ & $68.8 \%(22 / 32)$ & N.S. \\
Ongoing pregnancy rate & $41.7 \%(5 / 12)$ & $53.1 \%(17 / 32)$ & N.S. \\
\hline
\end{tabular}


Table 6. Characteristics of patients enrolled in single blastocyst transfer

\begin{tabular}{|c|c|c|c|}
\hline & Sequential $(n=12)$ & Single $(n=24)$ & $\mathbf{P}$ \\
\hline $\begin{array}{l}\text { Mean } \pm S D \text { age (year) } \\
\text { Age range (year) }\end{array}$ & $37.9 \pm 2.7(33-44)$ & $37.0 \pm 3.4(33-44)$ & N.S. \\
\hline $\mathrm{BMI}\left(\mathrm{kg} / \mathrm{m}^{2}\right)$ & $21.8 \pm 2.9(18.1-28.8)$ & $21.8 \pm 2.7(17.1-28.8)$ & N.S. \\
\hline $\mathrm{FSH}(\mathrm{IU} / \mathrm{ml})$ & $7.9 \pm 2.4(5.2-14.7)$ & $7.6 \pm 1.3(5.2-10.3)$ & N.S. \\
\hline Gravity & $0.83 \pm 0.90(3-0)$ & $0.71 \pm 0.93(3-0)$ & N.S. \\
\hline Parity & $0.58 \pm 0.64(2-0)$ & $0.17 \pm 0.37(1-0)$ & $<0.05$ \\
\hline $\begin{array}{l}\text { Period of fertility treatment } \\
\text { (month) }\end{array}$ & $17.3 \pm 13.8(3-96)$ & $25.6 \pm 23.7(3-96)$ & N.S. \\
\hline \multirow{6}{*}{ Cause of infertility } & Male factor: 5 & Male factor: 5 & \\
\hline & Tubal factor: 3 & Tubal factor: 8 & \\
\hline & Endometriosis: 1 & Endometriosis: 5 & \\
\hline & Ovulation disorder: 1 & Ovulation disorder: 0 & \\
\hline & Fertilization disorder: 0 & Fertilization disorder: 1 & \\
\hline & Unknown: 4 & Unknown: 9 & \\
\hline \multirow{4}{*}{ Fertilization method } & Conventional: 9 & Conventional: 19 & \\
\hline & ICSI: 2 & ICSI: 4 & \\
\hline & TESE-ICSI: 0 & TESE-ICSI: 0 & \\
\hline & Split: 1 & Split: 1 & \\
\hline
\end{tabular}

SD: Standard Deviation; BMI: Body Mass Index

JAMSAT

Table 7. Outcomes in terms of pregnancy rate and ongoing pregnancy rate

\begin{tabular}{cccc}
\hline & Sequential Culture Media $(\mathbf{n = 1 2})$ & Single Culture Medium $(\mathbf{n = 2 4 )}$ & $\mathbf{P}$ \\
\hline Pregnancy rate & $50.0 \%(6 / 12)$ & $66.7 \%(16 / 24)$ & N.S. \\
Ongoing pregnancy rate & $41.7 \%(5 / 12)$ & $54.2 \%(13 / 24)$ & N.S. \\
\hline
\end{tabular}

groups, although patient age and parity were significantly different (Table 4). No differences was observed in clinical pregnancy rates $(50.0 \%, 6 / 12$ vs. $68.8 \%$, $22 / 32 ; \mathrm{P}=0.30)$ or ongoing pregnancy rates $(41.7 \%, 5 / 12$ vs. $53.1 \%, 17 / 32 ; \mathrm{P}=0.74)$ in frozen-thawed blastocyst transfer between the sequential media culture and continuous single culture groups (Table 5). As eggs from younger individuals generally confer an advantage in achieving pregnancy, analysis of pregnancy and ongoing pregnancy rates were limited to 33 to 44-year-old patients. Although parity was still significantly different and the sequential media culture group possesses an advantage, age not differed (Table 6). No differences in clinical pregnancy rates $(50.0 \%, 6 / 12$ vs. $66.7 \%, 16 / 24$; $\mathrm{P}=0.47)$ or ongoing pregnancy rates $(41.7 \% 5 / 12$ vs. $54.2 \%, 13 / 24 ; \mathrm{P}=0.72$ ) were identified (Table 7).

\section{Discussion}

In the present study, continuous single medium culture was indicated to be better than sequential media culture regarding embryo development to the blastocyst stage in vitro. The formation rate of early cleavage embryos and their quality did not differ between the 2 culture media systems. However, embryos cultured in single medium showed an advanced blastulation rate and better quality among sibling embryos. Clinical and ongoing pregnancy rates did not differ between groups. These results suggest single continuous medium culture better than sequential media culture for achieving pregnancy with IVF, as single continuous medium produced a greater number of blastocysts with no different characteristics from those produced using sequential media. 
The advantage of embryo development in continuous single medium culture compared with sequential media culture may be due to the absence of medium renewal. A continuous single medium could avoid introduction of contamination through handling errors, decreased fluctuations in the culture environment including temperature and $\mathrm{pH}$, or decreased stress including visible light. As the differences in temperature and $\mathrm{pH}$ between sequential media are kept to a minimum, the main concern of sequential media may be the additional exposure to visible light. The handling of oocytes and embryos in vitro causes exposure to visible light (400 to $700 \mathrm{~nm}$ ), from the microscope and from ceiling lighting [19].

Visible light was reported to exert detrimental effects on embryo development. The negative effects on embryos are influenced by the period and intensity of exposure and the spectral composition of the light [20-22]. The usage of low illumination levels (100 Lux from the microscope, 20 Lux from ambient light) during human embryo manipulation throughout the IVF-embryo transfer in 110 IVF cycles were reported to yield a relatively high blastulation rate [21]. The effects of light on embryo development in mice and hamsters were also estimated by comparison between warm white fluorescent light with reduced levels of short-wavelength visible light and cool white fluorescent light, rich in short-wavelength visible light and commonly used in research and clinical laboratories. Mouse embryos cultured under shielding from light developed the most term fetuses, followed by those cultured under exposure to warm white fluorescent light, and then, cultured under cool white fluorescent light. It was reported to be due to greater production of reactive oxygen species while exposing to cool white fluorescent light [23]. Based on these facts, some component of visible light is thought to generate excessive detrimental reactive oxygen species, resulting in negative impacts on embryo development.

The other reason of advantage in the continuous single medium culture may be the specific amino acid composition of global tota ${ }^{\circledR}$ medium, a continuous single medium used in the present study. This medium contains all 20 Amino Acids (AAs), whereas ECM, sequential media used in the present study, contains only alanyl-glutamine. A measurable turnover of 17 AAs were shown in human early cleavage stage embryos [24]. Given the complexity of early embryo metabolism in vitro, usefulness or uselessness of AAs to the embryo still remained unclear. However, some AAs in media were reported to support embryo development to the blastocyst stage. Glycine and betaine are known to modulate osmolality deleteriously affected by high levels of inorganic ions [25].
Methionine is important for the synthesis of glutamine, hypotaurine, and taurine and was also suggested to be related to the Met-tRNA methylation of DNA [26]. On the other hand, the use of glutamine causes ammonium accumulation in the medium $[1,6]$. Accumulation of ammonium and metabolites of AAs in media is thought to disturb the developmental ability of embryo in culture media. Sequential media are superior in this respect, as medium renewal prevents such accumulations. However, instead of glutamine, the use of low concentrations L-alanyl-L-glutamine or glycyl-L-glutamine, stable forms of glutamine in media, had no effects on embryo development [27]. Taken together, the multiple AAs in commercial culture media appear to have more beneficial effects than adverse effects.

Embryo culture without medium renewal could reduce the cost of equipment and labor, and the time burdens on medical staff. As single medium was as good as or better than sequential media in terms of embryo development and pregnancy rate, the use of continuous single medium culture without medium renewal appears advantageous.

\section{Conclusion:}

The present study suggested the advantage of a single continuous culture system for infertile females as well as younger donors, as previously reported. A continuous single medium appeared superior for culturing human embryos in terms of blastulation rate, although the formation rate of early cleavage embryos, as well as pregnancy and ongoing pregnancy rates were not significantly different. These data suggested that single culture medium is as good as or better than a sequential media system for achieving pregnancy.

\section{Acknowledgements}

This research did not receive any specific grant from funding agencies in the public, commercial, or not-forprofit sectors.

\section{Conflict of Interest}

Each author's role is as follows: Akiko Takashima: Participant recruitment, performing IVF procedures, statistical analyses, and drafting of the manuscript; Fuminori Kimura: Study concept preparation, study design, interpreted analytical data, and reviewing and revision of the manuscript; Kazumi Kishida and Mika Izuno: Performing laboratory works for IVF and statistical analyses; Kimiko Hirata and Akie Takebayashi: Participant recruitment and performing IVF procedures; Tsukuru 
Amano, Shunichiro Tsuji, Shoji Kaku and Kentaro Takahashi: Contributed equally to data analysis; Tesuo Ono and Takashi Murakmai was involved in draft advice. The authors declared no conflict of interests.

\section{References}

[1] Chronopoulou E, Harper JC. IVF culture media: Past, present and future. Human Reproduction Update. 2014; 21(1):39-55. doi 10.1093/humupd/dmu040

[2] Wale PL, Gardner DK. The effects of chemical and physical factors on mammalian embryo culture and their importance for the practice of assisted human reproduction. Human Reproduction Update. 2015; 22(1):2-22. doi: 10.1093/humupd/dmv034

[3] Paternot G, Debrock S, D'Hooghe TM, Spiessens C. Early embryo development in a sequential versus single medium: A randomized study. Reproductive Biology and Endocrinology. 2010 8(1):83. doi: 10.1186/1477-7827-8-83

[4] Ciray HN, Aksoy T, Goktas C, Ozturk B, Bahceci M. Time-lapse evaluation of human embryo development in single versus sequential culture media: A sibling oocyte study. Journal of Assisted Reproduction and Genetics. 2012; 29(9):891-900. doi: 10.1007/ s10815-012-9818-7

[5] Gardner DK, Lane M. Towards a single embryo transfer. Reproductive Biomedicine Online. 2003; 6(4):470-81. doi: 10.1016/s14726483(10)62170-0

[6] Lane M, Gardner DK. Embryo culture medium: Which is the best. Best Practice \& Research Clinical Obstetrics \& Gynaecology. 2007; 21(1):83-100. doi: 10.1016/j.bpobgyn.2006.09.009

[7] Biggers JD, Summers MC. Choosing a culture medium: Making informed choices. Fertility and Sterility. 2008; 90(3):473-83. doi: 10.1016/j.fertnstert.2008.08.010

[8] Gardner DK, Lane M. Culture and selection of viable blastocysts: A feasible proposition for human IVF. Human Reproduction Update. 1997;3(4):367-82. doi: 10.1093/humupd/3.4.367

[9] Pool TB. An update on embryo culture for human assisted reproductive technology: Media, performance, and safety. Seminars in Reproductive Medicine. 2005; 23(4):309-18. doi: 10.1055/s-2005923388

[10] Biggers JD. Thoughts on embryo culture conditions. Reproductive Biomedicine Online. 2002; 4:30-8. doi: 10.1016/S14726483(12)60009-1

[11] Summers MC, Biggers JD. Chemically defined media and the culture of mammalian preimplantation embryos: Historical perspective and current issues. Human Reproduction Update. 2003 9(6):557-82. doi: 10.1093/humupd/dmg039

[12] Biggers JD, McGinnis LK, Lawitts JA. One-step versus two-step culture of mouse preimplantation embryos: Is there a difference. Human Reproduction. 2005; 20(12):3376-84. doi: 10.1093/humrep/dei228

[13] Mantikou E, Youssef MA, van Wely M, van der Veen F, Al Inany HG, Repping S, et al. Embryo culture media and IVF/ICSI success rates: A systematic review. Human Reproduction Update. 2013; 19(3):210-20. doi: 10.1093/humupd/dms061
[14] Ottosen LD, Hindkjær J, Ingerslev J. Light exposure of the ovum and preimplantation embryo during ART procedures. Journal of Assisted Reproduction and Genetics. 2007; 24(2-3):99-103. doi: 10.1007/s10815-006-9081-x

[15] Reed ML, Hamic A, Thompson DJ, Caperton CL. Continuous uninterrupted single medium culture without medium renewal versus sequential media culture: A sibling embryo study. Fertility and Sterility. 2009; 92(5):1783-6. doi: 10.1016/j.fertnstert.2009.05.008

[16] Sepúlveda S, Garcia J, Arriaga E, Diaz J, Noriega Portella L, Noriega Hoces L. In vitro development and pregnancy outcomes for human embryos cultured in either a single medium or in a sequential media system. Fertility and Sterility. 2009; 91(5):1765-70. doi: 10.1016/j.fertnstert.2008.02.16

[17] Wirleitner B, Vanderzwalmen P, Stecher A, Zech MH, Zintz M, Zech NH. Individual demands of human embryos on IVF culture medium: Influence on blastocyst development and pregnancy outcome. Reproductive Biomedicine Online. 2010; 21(6):776-82 doi: 10.1016/j.rbmo.2010.08.003

[18] Veeck LL. Oocyte assessment and biological performance. Annals of the New York Academy of Sciences. 1988; 541(1):259-74. doi: 10.1111/j.1749-6632.1988.tb22263.x

[19] Evers CA, Starr L. Biology: Concepts and applications. United States: Thomson; 2006.

[20] Takahashi M, Saka N, Takahashi H, Kanai Y, Schultz RM, Okano A. Assessment of DNA damage in individual hamster embryos by comet assay. Molecular Reproduction and Development. 1999; 54(1):1-7. doi: 10.1002/(sici)1098-2795(199909)54:1<1::aid$\operatorname{mrd1}>3.0 . \mathrm{co} ; 2-0$

[21] Umaoka Y, Noda Y, Nakayama T, Narimoto K, Mori T, Iritani A. Effect of visual light on in vitro embryonic development in the hamster. Theriogenology. 1992; 38(6):1043-54. doi: 10.1016/0093-691X(92)90118-B

[22] Schumacher A, Fischer B. Influence of visible light and room temperature on cell proliferation in preimplantation rabbit embryos. Journal of Reproduction and Fertility. 1988; 84(1):197-204 doi: 10.1530/jrf.0.0840197

[23] Takenaka M, Horiuchi T, Yanagimachi R. Effects of light on development of mammalian zygotes. National Academy of Sciences. 2007; 104(36):14289-93. doi: 10.1073/pnas.0706687104

[24] Houghton1 FD, Hawkhead JA, Humpherson PG, Hogg JE, Balen AH, Rutherford AJ, et al. Non-invasive amino acid turnover predicts human embryo developmental capacity. Human Reproduction. 2002; 17(4):999-1005. doi: 10.1093/humrep/17.4.999

[25] Van Winkle LJ, Haghighat N, Campione AL. Glycine protects preimplantation mouse conceptuses from a detrimental effect on development of the inorganic ions in oviductal fluid. Journal of Experimental Zoology Part A: Ecological Genetics and Physiology. 1990; 253(2):215-9. doi: 10.1002/jez.1402530211

[26] Ménézo Y, Lichtblau I, Elder K. New insights into human preimplantation metabolism in vivo and in vitro. Journal of Assisted Reproduction and Genetics. 2013; 30(3):293-303. doi: 10.1007/ s10815-013-9953-9

[27] Biggers JD, McGinnis LK, Lawitts JA. Enhanced effect of glycylL-glutamine on mouse preimplantation embryos in vitro. Reproductive Biomedicine Online. 2004; 9(1):59-69. doi: 10.1016/s14726483(10)62111-6 